\title{
Challenges to accurate food allergen labelling in food service outlets
}

\author{
C.M. Donovan ${ }^{1}$, C.B. O’Donovan ${ }^{1}$, M. Porter ${ }^{1}$, V. Cooling ${ }^{1}$, S. O’Mahony ${ }^{1}$, O.C. Lyons ${ }^{1,2}$, \\ C.J. Geraghty ${ }^{1}$, S. Taleghani ${ }^{1}$ and M.A.T. Flynn ${ }^{1,2}$ \\ ${ }^{1}$ Food Safety Authority of Ireland, Dublin 1, Republic of Ireland and ${ }^{2}$ Nutrition Innovation Centre for Food and \\ Health, Ulster University, Coleraine, BT52 ISA, UK.
}

Allergies affect $5-8 \%$ of children and $2-3 \%$ of adults in Ireland - most of these individuals suffer mild reactions while a small minority are affected by anaphylaxis which can be fatal ${ }^{(1)}$. Under food law introduced in December 2014, it is mandatory to provide information on 14 categories of allergens in non-prepacked food ${ }^{(2)}$. Providing accurate allergen information is particularly challenging for food service outlets (FSOs) for the following reasons - lack of knowledge due to no previous requirement to label food, continuous evolution of recipes and ingredients, and the presence of one or more of the 14 categories of allergens in most ingredients used. To help FSOs comply with the legislation, the Food Safety Authority of Ireland (FSAI) developed MenuCal ${ }^{(3)}$, a computerised system, that easily accommodates recipe modifications and ingredient changes. The aim of this study was to evaluate the implementation of allergen labelling and to identify what additional supports are required.

A targeted audit was conducted in November 2016 in line with the principles of the FSAI Audit Charter ${ }^{(4)}$. FSOs ( $\left.n 50\right)$, including hotels, restaurants, cafes, takeaways and pubs, were randomly selected across different regions in Ireland. In all cases no prior notice of the audit was given to FSOs. Compliance was assessed in terms of requirements outlined in the legislation i.e. allergen information in writing, easily accessible and visible to the consumer before the supply or sale of all foods, in legible script clearly indicating which allergens are present in which foods. The audit findings were communicated to Environmental Health Officers (EHOs) being offered training sessions on allergen food labelling.

\begin{tabular}{|c|c|c|c|}
\hline \multirow[b]{2}{*}{ Total FSOs } & \multicolumn{2}{|c|}{ Allergen labelling in place } & \multirow[b]{2}{*}{ Allergen labelling accurate } \\
\hline & Absent & Present & \\
\hline $100(50)$ & $32(16)$ & $68(34)$ & $16(8)$ \\
\hline Incorrect allergen labelling - main inaccuracies & & & $\%(\mathrm{n})$ \\
\hline Incorrect and/or incomplete allergen information & & & $48(24)$ \\
\hline Allergen information completely absent & & & $32(16)$ \\
\hline Allergen 'sulphites' missing & & & $22(11)$ \\
\hline Cereals containing gluten not specified & & & $20(10)$ \\
\hline Type of nut not specified & & & $20(10)$ \\
\hline Allergen information missing from alcoholic drinks & & & $16(8)$ \\
\hline Milk incorrectly specified as 'lactose' or 'dairy' & & & $8(4)$ \\
\hline Pine nuts listed as an allergen & & & $6(3)$ \\
\hline Allergen information missing on cereals containing gluten & & & $6(3)$ \\
\hline Crustaceans incorrectly specified as 'fish' & & & $4(2)$ \\
\hline Fish incorrectly specified as 'molluscs' or 'crustacean' & & & $4(2)$ \\
\hline Confusion over lupin & & & $4(2)$ \\
\hline
\end{tabular}

FSOs, food service outlets

Findings from the study showed that nearly a third of FSOs had no allergen labelling in place - reasons given included 'lack of time', 'unaware of the legal requirements', 'unsure how to identify allergens'. Only $16 \%$ of FSOs audited declared accurate allergen information and the sources of inaccuracies identified are listed in the table above. None of the FSOs were aware of the existence of MenuCal or that it is freely available. Following communication of the audit findings, eight allergen training sessions were undertaken to accommodate high numbers $(90 \%)$ of EHOs attending.

This study shows that accuracy of food allergen labelling is poor, putting vulnerable consumers at risk. The high attendance of EHOs at training sessions on allergens reflected the challenging nature of this legislation. On-going programmes to raise awareness among FSOs about their legal obligations and resources, such as MenuCal, is required to overcome the challenges involved in providing accurate information on allergens present in non-prepacked food.

1. INDI (2018) Food Allergies and Intolerances Fact Sheet https://www.indi.ie/diseases,-allergies-and-medical-conditions/food-allergy-and-intolerance (accessed March 2018).

2. S.I. No. 489/2014 - Health (Provision of Food Allergen Information to Consumers in respect of Non-Prepacked Food) Regulations (2014).

3. The Food Safety Authority of Ireland (2014) MenuCal. www.MenuCal.ie (Accessed March 2018).

4. The Food Safety Authority of Ireland (2017) Targeted Audit of Allergen Information on Non-Prepacked Food. https://www.fsai.ie/enforcement_audit/audit/reports.html (Accessed March 2018). 